\title{
Dynamical Descalarization in Binary Black Hole Mergers
}

\author{
Hector O. Silva $\oplus^{1,2,{ }^{*}}$ Helvi Witek $\oplus^{2, \dagger}$ Matthew Elley $\oplus^{3,+}$ and Nicolás Yunes $\oplus^{2, \S}$ \\ ${ }^{1}$ Max-Planck-Institut für Gravitationsphysik (Albert-Einstein-Institut), Am Mühlenberg 1, D-14476 Potsdam, Germany \\ ${ }^{2}$ Illinois Center for Advanced Studies of the Universe and Department of Physics, University of Illinois at Urbana-Champaign, \\ Urbana, Illinois 61801, USA \\ ${ }^{3}$ Department of Physics, King's College London, Strand, London WC2R 2LS, United Kingdom
}

(Received 23 December 2020; revised 16 March 2021; accepted 17 June 2021; published 14 July 2021)

Scalar fields coupled to the Gauss-Bonnet invariant can undergo a tachyonic instability, leading to spontaneous scalarization of black holes. Studies of this effect have so far been restricted to single black hole spacetimes. We present the first results on dynamical scalarization in head-on collisions and quasicircular inspirals of black hole binaries with numerical relativity simulations. We show that black hole binaries can either form a scalarized remnant or dynamically descalarize by shedding off its initial scalar hair. The observational implications of these findings are discussed.

DOI: 10.1103/PhysRevLett.127.031101

Introduction.-Despite the elegance of Einstein's theory, it presents several shortcomings: explaining the late-time acceleration of the Universe and providing a consistent theory of quantum gravity or the presence of spacetime singularities [e.g., in black holes (BHs)]. Candidate theories (of quantum gravity) that remedy these shortcomings typically predict the coupling to additional fields or higher curvature corrections [1]. Binary BHs, their gravitational wave $(\mathrm{GW})$ emission, and the first GW detections by the LIGO-Virgo Collaboration [2,3] offer unique insights into the nonlinear regime of gravity that unfolds during the BHs' inspiral and merger and enable new precision tests of gravity $[4,5]$. So far, these tests have been parametrized null tests against general relativity (GR) [6,7] or used a mapping between these parameters and those of specific theories [8-10]. To do the latter, however, requires GW predictions in specific theories.

One of the most compelling beyond-GR theories, scalar Gauss-Bonnet (SGB) gravity introduces a dynamical scalar field coupled to the Gauss-Bonnet invariant. SGB gravity emerges in the low-energy limit of quantum gravity paradigms such as string theory [11], through a dimensional reduction of Lovelock gravity [12] and is the simplest model that contains higher curvature operators. The most studied class of SGB gravity with a dilatonic or linear coupling to the scalar field gives rise to hairy BHs [13-19]. This theory, however, has been strongly constrained with GW observations from binary BHs [9].

Published by the American Physical Society under the terms of the Creative Commons Attribution 4.0 International license. Further distribution of this work must maintain attribution to the author(s) and the published article's title, journal citation, and DOI. Open access publication funded by the Max Planck Society.
We turn our attention to another interesting class of SGB gravity that is both unconstrained by GW observations and gives rise to (spontaneously) scalarized BHs [20,21]. Spontaneous scalarization is a familiar concept in beyond-GR theories; e.g., it is well established for neutron stars in scalar-tensor theories [22,23]. In such theories, the neutron star matter itself can induce a tachyonic instability that spontaneously scalarizes the star. When placed in a binary system, initially unscalarized neutron stars can scalarize dynamically near their merger or a scalarized neutron star can induce a scalar field in their unscalarized companion [24-27]. In SGB gravity, it is the spacetime curvature itself that induces scalarization of $\mathrm{BHs}[20,21]$, although this has only been shown for isolated BHs so far. In this Letter, we investigate, for the first time, dynamical scalarization in binary BHs. We concentrate on head-on collisions of $\mathrm{BHs}$, but also present the first binary $\mathrm{BH}$ inspiral study. Before doing so, it is convenient to first review the basics of SGB gravity and spontaneous BH scalarization.

Scalar Gauss-Bonnet gravity and scalarization.-SGB gravity is described by the action

$S=\frac{1}{16 \pi} \int d^{4} x \sqrt{-g}\left[R-\frac{1}{2}(\nabla \Phi)^{2}+\frac{\alpha_{\mathrm{GB}}}{4} f(\Phi) \mathcal{G}\right]$,

where a real scalar field $\Phi$ is coupled to the Gauss-Bonnet invariant $\mathcal{G}=R^{2}-4 R_{\mu \nu} R^{\mu \nu}+R_{\mu \nu \rho \sigma} R^{\mu \nu \rho \sigma}$, through the function $f(\Phi)$ and a dimensionful coupling constant $\alpha_{\mathrm{GB}}$. We use geometrical units $c=1=G$, in which $\alpha_{\mathrm{GB}}$ has units of $[\text { length }]^{2}$. The action (1) gives rise to the scalar field equation of motion

$$
\square \Phi=-\left(\alpha_{\mathrm{GB}} / 4\right) f^{\prime}(\Phi) \mathcal{G}
$$


where we defined $(\cdot)^{\prime}=d(\cdot) / d \Phi$. The function $f(\Phi)$ selects different "flavors" of SGB gravity $[28,29]$. One subset of these theories has $f^{\prime} \neq 0$ everywhere. It includes variants of SGB gravity with dilatonic $f(\Phi) \propto \exp (\Phi)$ [13-15] or shift-symmetric $f(\Phi) \propto \Phi[17,18,30]$ couplings, in which BHs always have scalar hair [19,31]. Another interesting class of SGB theories admits an extremum $f^{\prime}\left(\Phi_{0}\right)=0$ for a constant $\Phi_{0}$. They give rise to an effective space-dependent mass term $m_{\text {eff }}^{2}=$ $-f^{\prime \prime}\left(\Phi_{0}\right) \mathcal{G}$. This class includes quadratic $f(\Phi) \propto \Phi^{2}$ [21,32] and Gaussian $f(\Phi) \propto \exp \left(\Phi^{2}\right)$ [20] models.

The latter class still admits all vacuum $(\mathrm{BH})$ solutions of GR together with $\Phi=\Phi_{0}=$ const. In fact, if $f^{\prime \prime}\left(\Phi_{0}\right) \mathcal{G}<0$ these solutions are unique due to a no-hair theorem [21]. A linear stability study of these $\Phi_{0}=$ const solutions around a Schwarzschild BH reveals that this condition is a requirement for the absence of a tachyonic instability $\left(m_{\text {eff }}^{2}>0\right)$ for the scalar field perturbations [21]. If the effective mass $m_{\text {eff }}^{2}<0$, a tachyonic instability is triggered and the SGB scalar field is excited and spontaneously scalarizes the BHs. This linear instability [33] is quenched at the nonlinear level, resulting in a scalarized $\mathrm{BH}$ as end state [34]. The simplest theory that admits scalarized $\mathrm{BHs}$ is described by the quadratic coupling $f(\Phi)=\bar{\beta}_{2} \Phi^{2}$, where $\bar{\beta}_{2}=$ const [35]. The relevant parameter in this theory is the dimensionless constant $\beta_{2}=\left(\alpha_{\mathrm{GB}} / m^{2}\right) \bar{\beta}_{2}$, where $m$ is the characteristic mass of the system.

The onset of scalarization is fully determined by the scalar's linear dynamics on a given GR background. For a Schwarzschild BH of mass $m$, for which $\mathcal{G} \geq 0$ everywhere, scalarization first occurs for a spherically symmetric scalar field if $\beta_{2}=\beta_{c} \sim 1.45123$, a result in agreement with nonlinear calculations $[20,21]$. For values below $\beta_{c}$, the scalar perturbation decays monotonically at late times (we call them "subcritical"), precisely at $\beta_{c}$ the scalar field forms a bound state around the BH ("critical"), and above it the scalar field growths exponentially with time ("supercritical"). This result was recently generalized to Kerr BHs, where spin-induced scalarization can take place for $\beta_{2}<0$, for dimensionless spin parameters $\chi \geq 0.5$ [36-39]. Nonlinear rotating scalarized BH solutions in SGB gravity were found for both positive $[40,41]$ and negative $[42,43]$ values of $\beta_{2}$. So far studies of scalarization in SGB gravity focused on single BHs. We advance these studies to $\mathrm{BH}$ binaries and expand upon [44], focusing on the quadratic theory $f(\bar{\Phi})=\bar{\beta}_{2} \Phi^{2}$, as discussed next.

Numerical methods and simulations.-We investigate BH scalarization in the decoupling limit, i.e., we numerically evolve the scalar field on a time-dependent background in vacuum GR that represents binary $\mathrm{BH}$ spacetimes. Unless stated otherwise, we follow the approach of [44] and refer to it for details. We foliate the spacetime into spatial hypersurfaces with 3-metric $\gamma_{i j}$ and extrinsic curvature $K_{i j}=-(2 \alpha)^{-1} d_{t} \gamma_{i j}$, where $d_{t}=\partial_{t}-\mathcal{L}_{\beta}$, with $\mathcal{L}_{\beta}$ being the Lie derivative along the shift vector $\beta^{i}$, and $\alpha$ is the lapse function. We write Einstein's equations as a Cauchy problem and adopt the Baumgarte-Shapiro-Shibata-Nakamura formulation $[45,46]$ of the time evolution equations complemented with the moving-puncture gauge conditions $[47,48]$. We prepare Brill-Lindquist initial data $[49,50]$ for head-on collisions or Bowen-York initial data [51,52] for a quasicircular BH binary.

To evolve the scalar field, we introduce its momentum $K_{\Phi}=-\alpha^{-1} d_{t} \Phi$ and write its field equation (2) as

$$
\begin{aligned}
d_{t} \Phi & =-\alpha K_{\Phi}, \\
d_{t} K_{\Phi} & =-D^{i} \alpha D_{i} \Phi-\alpha\left(D^{i} D_{i} \Phi-K K_{\Phi}+\frac{\alpha_{\mathrm{GB}}}{4} f^{\prime} \mathcal{G}\right),
\end{aligned}
$$

where $D_{i}$ is the covariant derivative associated with $\gamma_{i j}$, $K=\gamma^{i j} K_{i j}, f^{\prime}=2 \bar{\beta}_{2} \Phi$, and $\mathcal{G}$ is the Gauss-Bonnet invariant of the background spacetime. We set the system's total mass to unity, i.e., $M=m_{1}+m_{2}=1$, where $m=m_{1,2}$ is the component's mass. The scalar field is initialized either as a spherically symmetric Gaussian shell $(G)$, located at $r_{0}=12 M$ and with width $\sigma=1 M$ as in [44], or as a bound state $(B)$ around each binary component

$\left.\Phi\right|_{t=0}=\frac{m r}{\varrho^{2}}\left[c_{1}+\frac{c_{2} m r}{\varrho^{2}}+\frac{c_{3}(m r)^{2}}{\varrho^{4}}\right],\left.\quad K_{\Phi}\right|_{t=0}=0$.

Here, $Q=m+2 r$, and $c_{1}=3.68375, c_{2}=4.972416$, and $c_{3}=4.972416 \times 10^{2}$ are fitting constants to reproduce the numerical results in [21].

We perform our numerical simulations with Canuda [44,53-55], coupled to the open-source Einstein Toolkit $[56,57]$. We extended the implementation of [44] to general coupling functions $f$, including the quadratic coupling. We employ the method of lines with fourth-order finite difference stencils to realize spatial derivatives and a fourthorder Runge-Kutta time integrator. We use box-in-box mesh refinement provided by Carpet [58]. The numerical grid contains seven refinement levels, with the outer boundary located at $256 M$ and a grid spacing of $d x=$ $1.0 M$ on the outer mesh. To assess the numerical accuracy of our simulations, we evolved Fig. 1(b) with additional resolutions $d x=0.9 M$ and $d x=0.8 M$. We find secondorder convergence and a relative discretization error of $\Delta \Phi_{00} / \Phi_{00} \lesssim 0.5 \%$, where $\Phi_{00}$ is the $\ell=\mathfrak{m}=0$ multipole of the scalar field. We present the corresponding convergence plot for the scalar monopole and for the gravitational wave $l=2, \mathfrak{m}=0$ mode in Fig. 1 of the Supplemental Material [59].

Results.-We performed a large set of $\mathrm{BH}$ head-on collisions with varying mass ratio $q=m_{1} / m_{2} \leq 1$, total mass $M=1$, and initial separation $d=25 M$. The BHs merge at $t_{M} \sim 179.5 M$, as estimated from the peak of the 

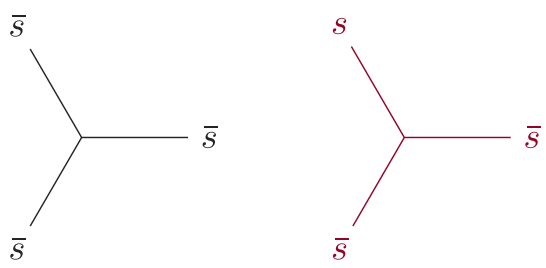

(a) $-\{\mathrm{G}, 1,0\}$

(b) $-\{\mathrm{B}, 1 / 2,0.16125\}$

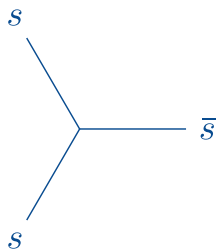

(c) $-\{\mathrm{B}, 1,0.36281\}$

FIG. 1. Summary of simulations of BH head-on collisions, where $\bar{s}$ and $s$ stand for initial or final states that are either nonscalarized or scalarized, respectively. Each diagram is labeled by the initial data (Gaussian shell " $G$ " or bound state " $B$ "), the mass ratio $q=m_{1} / m_{2}(1$ or $1 / 2)$, and the coupling parameter $\beta_{2}$. In case (a) (top left panel) two nonscalarized BHs produce a nonscalarized remnant. In case (b) (top right panel) a scalarized and a nonscalarized $\mathrm{BH}$ produce a nonscalarized remnant. This initial configuration is possible when $q$ is different from one. In case (c) (bottom left panel) two scalarized BHs produce a nonscalarized remnant. Finally, in case (d) (bottom right panel) two scalarized $\mathrm{BHs}$ produce a scalarized remnant.

$\ell=2, \mathfrak{m}=0$ multipole of the gravitational waveform. To guide our choices of $\beta_{2}$, we recall that the critical coupling for the fundamental mode is $\beta_{2, c}=\beta_{c}(m / M)^{2}$ with $\beta_{c} \sim 1.45123$, and $m$ denotes either the individual BH's mass $m_{1,2}$ or the total mass $M$. For example, for an equalmass binary with $m_{1}=m_{2}=M / 2$, the critical coupling for the individual holes is $\beta_{2, c}^{(1)}=\beta_{2, c}^{(2)}=\beta_{c} / 4=0.36275$ and that of the final hole is approximately $\beta_{2, c}^{f}=\beta_{c}$, where we neglected the small mass loss in the form of GWs during the collision $[60,61]$.

Here we present a selection of our results, illustrated in Fig. 1, to highlight our most important findings. An expanded discussion will be presented in a companion paper [62]. We vary the initial state by setting the coupling parameter $\beta_{2}$ such that [Fig. 1(a)] none of the BHs are initially scalarized, [Fig. 1(b)] the smaller-mass BH initially carries a bound-state scalar field, and both BHs carry initially a bound-state scalar that leads either to a nonscalarized final $\mathrm{BH}$ [Fig. 1(c)] or a scalarized final $\mathrm{BH}$ [Fig. 1(d)].

In Fig. 2 we show the $\ell=\mathfrak{m}=0$ scalar field multipole extracted on a sphere of fixed radius $r_{\mathrm{ex}}=50 \mathrm{M}$, as a function of time, and we present snapshots of the scalar's profile in the Supplemental Material [59]. In Fig. 1(a), the scalar perturbation is not supported at all (since $\left.m_{\mathrm{eff}}=0\right)$

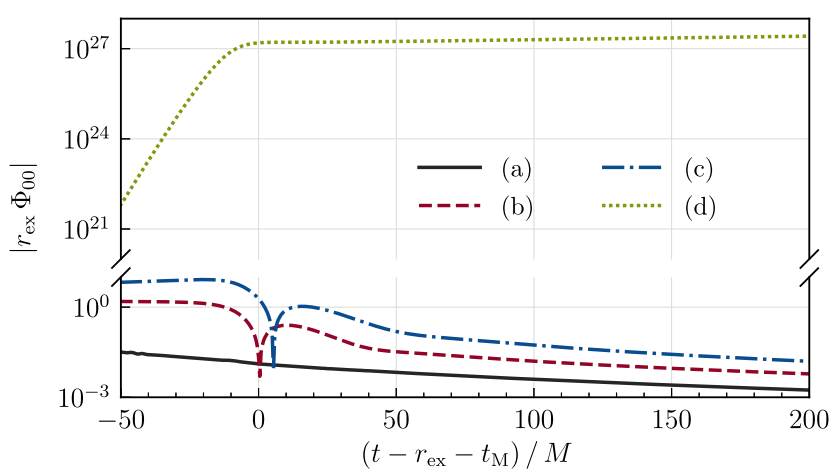

FIG. 2. Time evolution of the scalar field $\ell=\mathfrak{m}=0$ multipole in the background of a $\mathrm{BH}$ head-on collision with initial separation $d=25 M$. It is rescaled by the extraction radius $r_{\mathrm{ex}}=$ $50 M$ and shifted in time such that $\left(t-r_{\mathrm{ex}}-t_{M}\right) / M=0$ corresponds to the BHs' merger. The labels refer to the four cases summarized in Fig. 1.

and, indeed, after a brief interaction at early times it decays already before the BHs collide. In Figs. 1(b) and 1(c), we find a constant scalar field before the BHs collide that is consistent with a bound state around the individual $(q=1)$ or smaller-mass $\mathrm{BH}(q=1 / 2)$. After the merger, the scalar field decays since the curvature (and thus $m_{\text {eff }}$ ) decreases and the system no longer supports a bound state- the final BH dynamically descalarizes. In Fig. 1(d), the scalar field grows exponentially before the merger because it is supercritical for the individual BHs and settles to a constant in time that is consistent with a bound state around the final $\mathrm{BH}$.

In Fig. 3, we show two-dimensional snapshots of the scalar field and spacetime curvature for Fig. 1(b), which illustrates the dynamical descalarization phenomenon [63]. The color map is shared among all panels and shows the amplitude of $\log _{10}|\Phi|$, while the curves are isocurvature levels of $\mathcal{G} M^{4}=\left\{1,10^{-1}, 10^{-2}, 10^{-3}\right\}$. Initially, at $t=1 M$, each $\mathrm{BH}$ (whose locations are revealed by the isocurvature levels) are surrounded by nontrivial scalar field initial data given by Eq. (4). At $t=50 M$, the smaller $\mathrm{BH}$ hosts a bound state scalar that is dragged along the hole's motion, inducing scalar dipole radiation that would impact the GWs emitted. In contrast, the scalar field around the larger BH disperses because its curvature is too small to sustain a bound state for a coupling of $\beta_{2}=0.36281$. The system thus evolves as an $s+\bar{s}$ process in the notation of Fig. 1. At $t=160 \mathrm{M}$, the BHs are about to merge, as indicated by the two lobes in the isocurvature contours, the curvature of the combined system decreases, and the scalar field starts dissipating. At $t=182 M$, which is shortly after the collision, the system has descalarized since for the final $\mathrm{BH} \beta_{2, c}^{f}>\beta_{2}$.

We also simulated the inspiral of an equal-mass, nonspinning BH binary with initial separation of $d=10 \mathrm{M}$, $\beta_{2}=0.36281$, and bound state scalar field initial data. This 

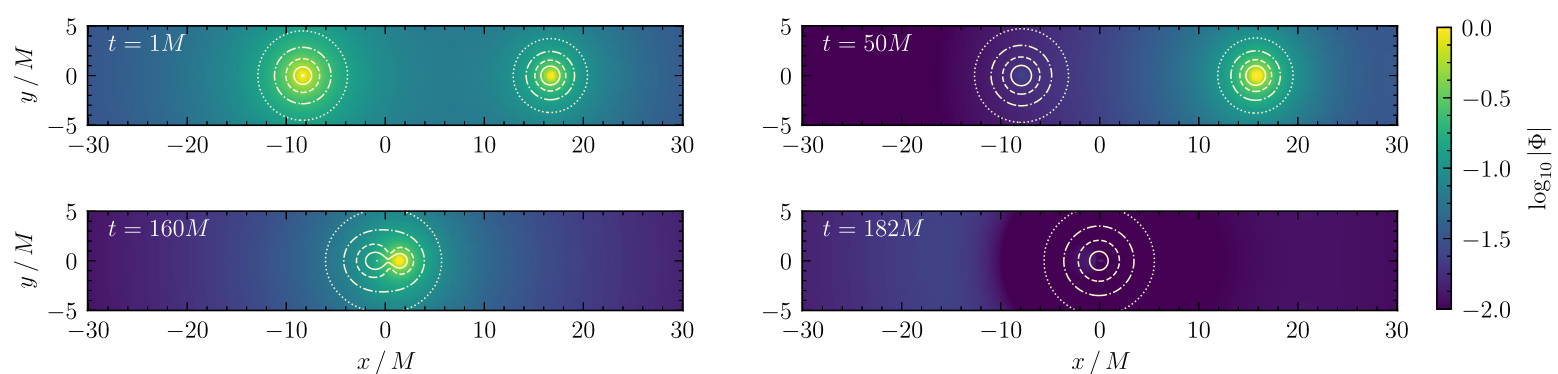

FIG. 3. Scalar field and Gauss-Bonnet dynamics on the $x y$ plane for Fig. 1(b). We show the amplitude of $\log _{10}|\Phi|$ (color map) together with the Gauss-Bonnet invariant (isocurvature levels) at the beginning of the evolution (top left), during the BHs' approach (top right), shortly before the collision (bottom left), and shortly after the merger (bottom right). The isocurvature levels correspond to $1 M^{-4}$ (solid line), $10^{-1} M^{-4}$ (dashed line), $10^{-2} M^{-4}$ (dot-dashed line), and $10^{-3} M^{-4}$ (dotted line).

corresponds to an initial configuration in which both BHs are scalarized, and then, after merger, the remnant is not scalarized, which is analogous to Fig. 1(c) in the head-on case. In Fig. 4, we show the gravitational quadrupole waveform (bottom panel), as characterized by the $\ell=$ $\mathfrak{m}=2$ mode of the Newman-Penrose scalar $\Psi_{4}$, together with the scalar field's monopole (top) and quadrupole (middle). The scalar's monopole $\Phi_{00}$ exhibits the distinctive signature of descalarization: the increase in the field's amplitude during the inspiral of scalarized BHs is followed by a complete dissipation of the scalar field after the merger $\left(t_{M} \sim 917 M\right)$ as the curvature of the remnant $\mathrm{BH}$ no longer supports a bound state. In addition, the dynamics of the BH binary sources scalar quadrupole radiation. The field's amplitude grows exponentially during the inspiral and decays after the BHs have merged. The origin of this excitation is not direct scalarization of the $\ell=2$ scalar bound state, but due to the inspiral of two scalarized (or
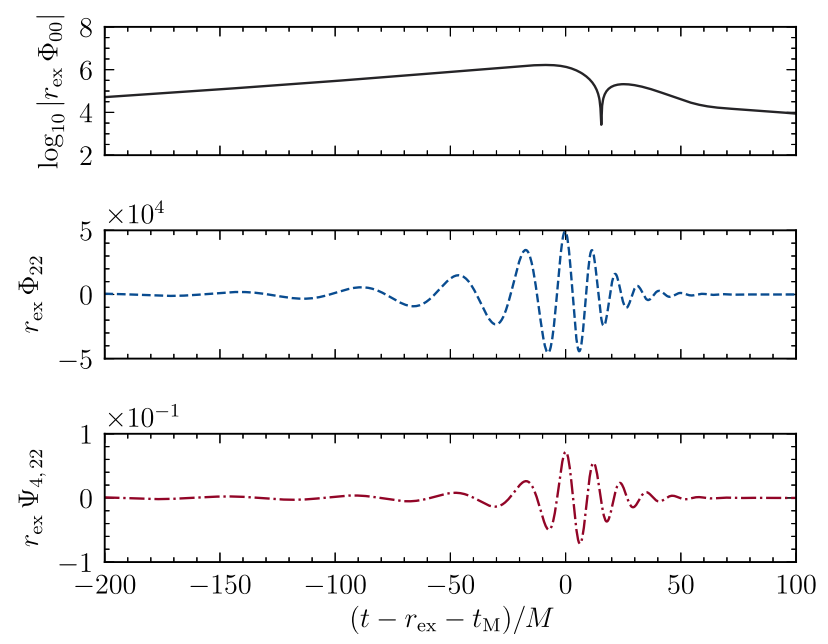

FIG. 4. Scalar and gravitational waveforms, rescaled by the extraction radius $r_{\mathrm{ex}}=50 \mathrm{M}$, sourced by an equal-mass $\mathrm{BH}$ binary with bound state initial data on each BH. This system is the inspiral counterpart of Fig. 1(c) and shows dynamical descalarization in action. "hairy") BHs. This interpretation is further supported by the observation that the phase of the $\ell=\mathfrak{m}=2$ scalar mode is driven by the binary's orbital frequency. We also observed this for the $\ell=\mathfrak{m}=4$ mode and expect it to happen for all even $\ell=\mathfrak{m}$ modes. For $q=1$, the odd $\ell=\mathfrak{m}$ modes are suppressed due to symmetry, whereas they would be excited in the general case $q \neq 1$. The descalarization during the merger is reminiscent of the decrease in scalar charge observed in the shift-symmetric theory [44], however, with the striking difference that here the remnant $\mathrm{BH}$ is a rotating GR solution.

Discussions. - We presented the first numerical relativity simulations of the scalar field dynamics in binary BH spacetimes in quadratic SGB gravity [21]. We found that the interplay between mass ratio $q$ and $\beta_{2}$ can result in different scenarios for the scalar field dynamics. Most notably, it can lead to a dynamical descalarization of the binary, which we observed in both head-on and quasicircular inspiral simulations. Here we focused on $\beta_{2} \geq 0$, but the case $\beta_{2}<0$ would be particularly interesting to study in inspiral simulations. More specifically, the spinning remnant of a binary BH merger typically has a dimensionless spin $\chi \sim 0.7$ [64], sufficient to trigger a spin-induced tachyonic instability of the scalar field [36]. This is currently under study [62]. It would be interesting to frame this effect within the effective field theory (EFT) of [65] or in a post-Newtonian framework [66-68].

The scalar excitations we have discovered during the binary $\mathrm{BH}$ coalescence in this class of SGB theories have important implications to GW observations and tests of GR. In particular, the scalar excitations will drain the binary of energy as they propagate away from the system, the monopole scalar piece inducing dipole losses, and the quadrupole piece correcting the quadrupole GW losses of GR, which, based on [69], are expected to only have the same "plus" and "cross" polarizations. This enhanced dissipation of energy and angular momentum, in turn, will force the binary to inspiral faster than in GR and, therefore, leave an imprint in the GWs emitted through corrections to the rate at which the GW frequency increases during the 
inspiral. This GW phase shift will enable us to project bounds on SGB gravity that are similar in spirit but complementary to the analysis of [44]. In fact, because the merger leaves behind a "bald" Kerr black hole due to dynamical descalarization, the (scalar) energy flux is, in general, larger as compared to shift-symmetric SGB, where the remnant black hole always retains some of its hair. This suggests that strong observational bounds might be placed on this theory. A detailed numerical analysis of this expected GW phase shift during of the late inspiral, merger, and ringdown will be presented in upcoming work [62].

Having worked in the decoupling limit, a question naturally arises: what would we expect in the fully nonlinear regime of SGB gravity? It is known that nonlinear effects set an upper bound on the scalar field magnitude at the $\mathrm{BH}$ horizon [28], so that the domain of existence of scalarized BHs exhibits a very narrow bandlike structure in the phase space spanned by $\mathrm{BH}$ mass and coupling $\beta_{2}$; see Fig. 2 of [21]. This means that Fig. 1(d) would only occur for sufficiently small mass ratios such that both the initial binary and its final state remain in band. In general, however, comparable mass $\mathrm{BH}$ binaries could undergo an $\bar{s}+\bar{s} \rightarrow s$ process, in which two unscalarized $\mathrm{BHs}$ would merge, forming a $\mathrm{BH}$ within the scalarization band. The descalarization of the $\mathrm{BH}$ remnant would also impact the GW emission during the ringdown. Specifically, the waveforms in Fig. 4 show that the ringdown timescales of scalar and tensorial modes are comparable. This suggests that one should expect to see the imprint of the descalarization onto the quasinormal mode spectra of the Kerr black hole in the nonlinear case. Performing these studies in practice would require a general, well-posed formulation of the time evolution equations outside the EFT approach [44,70], small values of the coupling parameter [71,72], or spherical symmetry [34,73-75]. Finding such a formulation has proven challenging [76-79], although first results in this direction were presented [80]. Our work motivates and paves the way for future studies of nonperturbative, beyond-GR effects in BH binaries, with potential implications to tests of GR with GW astronomy.

We thank Katy Clough, Mohammed Khalil, and Jan Steinhoff for useful discussions. H. W. acknowledges financial support provided by the NSF Grant No. OAC2004879, the Royal Society University Research Fellowship Grant No. UF160547, and the Royal Society Research Grant No. RGFIR1\180073. H. O. S and N. Y. acknowledge financial support through NSF Grants No. PHY-1759615 and No. PHY-1949838, and NASA ATP Grants No. 17-ATP17-0225, No. NNX16AB98G, and No. 80NSSC17M0041. We thankfully acknowledge the computer resources and the technical support provided by the Leibniz Supercomputing Center via PRACE Grant No. 2018194669 "FunPhysGW: Fundamental physics in the era of gravitational waves" and by the DiRAC Consortium via STFC DiRAC Grants No. ACTP186 and
No. ACSP218. This work made use of the Illinois Campus Cluster, a computing resource that is operated by the Illinois Campus ClusterProgram (ICCP) in conjunction with the National Center for Supercomputing Applications (NCSA) and which is supported by funds from the University of Illinois at Urbana-Champaign.

*hector.silva@aei.mpg.de

†hwitek@illinois.edu

†matthew.elley@kcl.ac.uk

§nyunes@illinois.edu

[1] E. Berti et al., Testing general relativity with present and future astrophysical observations, Classical Quantum Gravity 32, 243001 (2015).

[2] B. Abbott et al. (LIGO Scientific, Virgo Collaborations), GWTC-1: A Gravitational-Wave Transient Catalog of Compact Binary Mergers Observed by LIGO and Virgo during the First and Second Observing Runs, Phys. Rev. X 9, 031040 (2019).

[3] R. Abbott et al. (LIGO Scientific, Virgo Collaborations), GWTC-2: Compact Binary Coalescences Observed by LIGO and Virgo during the First Half of the Third Observing Run, Phys. Rev. X 11, 021053 (2021).

[4] N. Yunes and X. Siemens, Gravitational-wave tests of general relativity with ground-based detectors and pulsar timing-arrays, Living Rev. Relativity 16, 9 (2013).

[5] K. Yagi and L. C. Stein, Black hole based tests of general relativity, Classical Quantum Gravity 33, 054001 (2016).

[6] B. Abbott et al. (LIGO Scientific, Virgo Collaborations), Tests of general relativity with the binary black hole signals from the LIGO-Virgo catalog GWTC-1, Phys. Rev. D 100, 104036 (2019).

[7] R. Abbott et al. (LIGO Scientific, Virgo Collaborations), Tests of general relativity with binary black holes from the second LIGO-Virgo gravitational-wave transient catalog, Phys. Rev. D 103, 122002 (2021).

[8] N. Yunes, K. Yagi, and F. Pretorius, Theoretical physics implications of the binary black-hole mergers GW150914 and GW151226, Phys. Rev. D 94, 084002 (2016).

[9] R. Nair, S. Perkins, H. O. Silva, and N. Yunes, Fundamental Physics Implications for Higher-Curvature Theories from Binary Black Hole Signals in the LIGO-Virgo Catalog GWTC-1, Phys. Rev. Lett. 123, 191101 (2019).

[10] Z. Carson and K. Yagi, Testing general relativity with gravitational waves, arXiv:2011.02938.

[11] R. Metsaev and A. A. Tseytlin, Order $\alpha^{\prime}$ (two loop) equivalence of the string equations of motion and the sigma model Weyl invariance conditions: Dependence on the dilaton and the antisymmetric tensor, Nucl. Phys. B293, 385 (1987).

[12] C. Charmousis, From Lovelock to Horndeski's generalized scalar tensor theory, Lect. Notes Phys. 892, 25 (2015).

[13] S. Mignemi and N. R. Stewart, Charged black holes in effective string theory, Phys. Rev. D 47, 5259 (1993).

[14] P. Kanti, N. E. Mavromatos, J. Rizos, K. Tamvakis, and E. Winstanley, Dilatonic black holes in higher curvature string gravity, Phys. Rev. D 54, 5049 (1996). 
[15] T. Torii, H. Yajima, and K.-I. Maeda, Dilatonic black holes with Gauss-Bonnet term, Phys. Rev. D 55, 739 (1997).

[16] N. Yunes and L. C. Stein, Non-spinning black holes in alternative theories of gravity, Phys. Rev. D 83, 104002 (2011).

[17] T. P. Sotiriou and S.-Y. Zhou, Black Hole Hair in Generalized Scalar-Tensor Gravity, Phys. Rev. Lett. 112, 251102 (2014).

[18] T. P. Sotiriou and S.-Y. Zhou, Black hole hair in generalized scalar-tensor gravity: An explicit example, Phys. Rev. D 90, 124063 (2014).

[19] K. Prabhu and L. C. Stein, Black hole scalar charge from a topological horizon integral in Einstein-dilaton-GaussBonnet gravity, Phys. Rev. D 98, 021503(R) (2018).

[20] D. D. Doneva and S.S. Yazadjiev, New Gauss-Bonnet Black Holes with Curvature-Induced Scalarization in Extended Scalar-Tensor Theories, Phys. Rev. Lett. 120, 131103 (2018).

[21] H. O. Silva, J. Sakstein, L. Gualtieri, T. P. Sotiriou, and E. Berti, Spontaneous Scalarization of Black Holes and Compact Stars from a Gauss-Bonnet Coupling, Phys. Rev. Lett. 120, 131104 (2018).

[22] T. Damour and G. Esposito-Farèse, Nonperturbative Strong Field Effects in Tensor-Scalar Theories of Gravitation, Phys. Rev. Lett. 70, 2220 (1993).

[23] T. Damour and G. Esposito-Farèse, Tensor-scalar gravity and binary pulsar experiments, Phys. Rev. D 54, 1474 (1996).

[24] E. Barausse, C. Palenzuela, M. Ponce, and L. Lehner, Neutron-star mergers in scalar-tensor theories of gravity, Phys. Rev. D 87, 081506(R) (2013).

[25] C. Palenzuela, E. Barausse, M. Ponce, and L. Lehner, Dynamical scalarization of neutron stars in scalar-tensor gravity theories, Phys. Rev. D 89, 044024 (2014).

[26] M. Shibata, K. Taniguchi, H. Okawa, and A. Buonanno, Coalescence of binary neutron stars in a scalar-tensor theory of gravity, Phys. Rev. D 89, 084005 (2014).

[27] N. Sennett, L. Shao, and J. Steinhoff, Effective action model of dynamically scalarizing binary neutron stars, Phys. Rev. D 96, 084019 (2017).

[28] G. Antoniou, A. Bakopoulos, and P. Kanti, Evasion of No-Hair Theorems and Novel Black-Hole Solutions in Gauss-Bonnet Theories, Phys. Rev. Lett. 120, 131102 (2018).

[29] G. Antoniou, A. Bakopoulos, and P. Kanti, Black-hole solutions with scalar hair in Einstein-Scalar-Gauss-Bonnet theories, Phys. Rev. D 97, 084037 (2018).

[30] A. Maselli, P. Pani, L. Gualtieri, and V. Ferrari, Rotating black holes in Einstein-dilaton-Gauss-Bonnet gravity with finite coupling, Phys. Rev. D 92, 083014 (2015).

[31] K. Yagi, L. C. Stein, and N. Yunes, Challenging the presence of scalar charge and dipolar radiation in binary pulsars, Phys. Rev. D 93, 024010 (2016).

[32] C. F. B. Macedo, J. Sakstein, E. Berti, L. Gualtieri, H. O. Silva, and T.P. Sotiriou, Self-interactions and spontaneous black hole scalarization, Phys. Rev. D 99, 104041 (2019).

[33] C. F. Macedo, Scalar modes, spontaneous scalarization and circular null-geodesics of black holes in scalarGauss-Bonnet gravity, Int. J. Mod. Phys. D 29, 2041006 (2020).
[34] J. L. Ripley and F. Pretorius, Dynamics of a $\mathbb{Z}_{2}$ symmetric EdGB gravity in spherical symmetry, Classical Quantum Gravity 37, 155003 (2020).

[35] We can translate the notation used in [21] to that used here with the replacement $\eta=2 \alpha_{\mathrm{GB}} \bar{\beta}_{2}$.

[36] A. Dima, E. Barausse, N. Franchini, and T. P. Sotiriou, SpinInduced Black Hole Spontaneous Scalarization, Phys. Rev. Lett. 125, 231101 (2020).

[37] S. Hod, Onset of spontaneous scalarization in spinning Gauss-Bonnet black holes, Phys. Rev. D 102, 084060 (2020).

[38] D. D. Doneva, L. G. Collodel, C. J. Krüger, and S. S. Yazadjiev, Black hole scalarization induced by the spin: $2+$ 1 time evolution, Phys. Rev. D 102, 104027 (2020).

[39] D. D. Doneva and S. S. Yazadjiev, On the dynamics of the nonrotating and rotating black hole scalarization, Phys. Rev. D 103, 064024 (2021).

[40] P. V. P. Cunha, C. A. R. Herdeiro, and E. Radu, Spontaneously Scalarized Kerr Black Holes in Extended ScalarTensor-Gauss-Bonnet Gravity, Phys. Rev. Lett. 123, 011101 (2019).

[41] L. G. Collodel, B. Kleihaus, J. Kunz, and E. Berti, Spinning and excited black holes in Einstein-scalarGauss-Bonnet theory, Classical Quantum Gravity 37, 075018 (2020).

[42] C. A. R. Herdeiro, E. Radu, H. O. Silva, T. P. Sotiriou, and N. Yunes, Spin-Induced Scalarized Black Holes, Phys. Rev. Lett. 126, 011103 (2021).

[43] E. Berti, L. G. Collodel, B. Kleihaus, and J. Kunz, SpinInduced Black-Hole Scalarization in Einstein-ScalarGauss-Bonnet Theory, Phys. Rev. Lett. 126, 011104 (2021).

[44] H. Witek, L. Gualtieri, P. Pani, and T. P. Sotiriou, Black holes and binary mergers in scalar Gauss-Bonnet gravity: Scalar field dynamics, Phys. Rev. D 99, 064035 (2019).

[45] M. Shibata and T. Nakamura, Evolution of threedimensional gravitational waves: Harmonic slicing case, Phys. Rev. D 52, 5428 (1995).

[46] T. W. Baumgarte and S. L. Shapiro, On the numerical integration of Einstein's field equations, Phys. Rev. D 59, 024007 (1998).

[47] M. Campanelli, C. O. Lousto, P. Marronetti, and Y. Zlochower, Accurate Evolutions of Orbiting Black-Hole Binaries without Excision, Phys. Rev. Lett. 96, 111101 (2006).

[48] J. G. Baker, J. Centrella, D.-I. Choi, M. Koppitz, and J. van Meter, Gravitational Wave Extraction from an Inspiraling Configuration of Merging Black Holes, Phys. Rev. Lett. 96, 111102 (2006).

[49] D. R. Brill and R. W. Lindquist, Interaction energy in geometrostatics, Phys. Rev. 131, 471 (1963).

[50] R. W. Lindquist, Initial-Value Problem on Einstein-Rosen Manifolds, J. Math. Phys. (N.Y.) 4, 938 (1963).

[51] J. M. Bowen and J. W. York, Time asymmetric initial data for black holes and black hole collisions, Phys. Rev. D 21, 2047 (1980).

[52] S. Brandt and B. Bruegmann, A Simple Construction of Initial Data for Multiple Black Holes, Phys. Rev. Lett. 78, 3606 (1997). 
[53] H. Witek, M. Zilhão, G. Ficarra, and M. Elley, Canuda: A public numerical relativity library to probe fundamental physics (2020), http://doi.org/10.5281/zenodo.3565475.

[54] R. Benkel, T. P. Sotiriou, and H. Witek, Dynamical scalar hair formation around a Schwarzschild black hole, Phys. Rev. D 94, 121503(R) (2016).

[55] R. Benkel, T. P. Sotiriou, and H. Witek, Black hole hair formation in shift-symmetric generalised scalar-tensor gravity, Classical Quantum Gravity 34, 064001 (2017).

[56] S. R. Brandt et al., The Einstein Toolkit (2020), to find out more, visit http://doi.org/10.5281/zenodo.4884780.

[57] F. Löffler et al., The Einstein Toolkit: A community computational infrastructure for relativistic astrophysics, Classical Quantum Gravity 29, 115001 (2012).

[58] E. Schnetter, S. H. Hawley, and I. Hawke, Evolutions in 3-D numerical relativity using fixed mesh refinement, Classical Quantum Gravity 21, 1465 (2004).

[59] See Supplemental Material at http://link.aps.org/ supplemental/10.1103/PhysRevLett.127.031101 for convergence tests and snapshots of the scalar field spatial profile before, near and after the merger in our head-on simulations.

[60] C. O. Lousto and R. H. Price, Radiation content of conformally flat initial data, Phys. Rev. D 69, 087503 (2004).

[61] U. Sperhake, V. Cardoso, C. D. Ott, E. Schnetter, and H. Witek, Extreme black hole simulations: Collisions of unequal mass black holes and the point particle limit, Phys. Rev. D 84, 084038 (2011).

[62] M. Elley, H. Witek, H. O. Silva, and N. Yunes, Spontaneous scalarization in compact binaries in scalar-Gauss-Bonnet gravity (to be published).

[63] Videos of our simulations are available at https:// bhscalarization.bitbucket.io.

[64] A. Buonanno, G. B. Cook, and F. Pretorius, Inspiral, merger and ring-down of equal-mass black-hole binaries, Phys. Rev. D 75, 124018 (2007).

[65] M. Khalil, N. Sennett, J. Steinhoff, and A. Buonanno, Theory-agnostic framework for dynamical scalarization of compact binaries, Phys. Rev. D 100, 124013 (2019).

[66] K. Yagi, L. C. Stein, N. Yunes, and T. Tanaka, Post-Newtonian, quasi-circular binary inspirals in quadratic modified gravity, Phys. Rev. D 85, 064022 (2012); Erratum, Phys. Rev. D 93, 029902 (2016).
[67] F.-L. Julié and E. Berti, Post-Newtonian dynamics and black hole thermodynamics in Einstein-scalar-Gauss-Bonnet gravity, Phys. Rev. D 100, 104061 (2019).

[68] B. Shiralilou, T. Hinderer, S. Nissanke, N. Ortiz, and H. Witek, Nonlinear curvature effects in gravitational waves from inspiralling black hole binaries, Phys. Rev. D 103 L121503 (2021).

[69] P. Wagle, A. Saffer, and N. Yunes, Polarization modes of gravitational waves in quadratic gravity, Phys. Rev. D 100, 124007 (2019).

[70] M. Okounkova, Numerical relativity simulation of GW150914 in Einstein dilaton Gauss-Bonnet gravity, Phys. Rev. D 102, 084046 (2020).

[71] A. D. Kovács and H. S. Reall, Well-posed formulation of Lovelock and Horndeski theories, Phys. Rev. D 101, 124003 (2020).

[72] A. D. Kovács and H. S. Reall, Well-Posed Formulation of Scalar-Tensor Effective Field Theory, Phys. Rev. Lett. 124, 221101 (2020).

[73] J. L. Ripley and F. Pretorius, Hyperbolicity in spherical gravitational collapse in a Horndeski theory, Phys. Rev. D 99, 084014 (2019).

[74] J. Ripley and F. Pretorius, Gravitational collapse in Einstein dilaton-Gauss-Bonnet gravity, Classical Quantum Gravity 36, 134001 (2019).

[75] J. L. Ripley and F. Pretorius, Scalarized black hole dynamics in Einstein dilaton Gauss-Bonnet gravity, Phys. Rev. D 101, 044015 (2020).

[76] G. Papallo and H. S. Reall, On the local well-posedness of Lovelock and Horndeski theories, Phys. Rev. D 96, 044019 (2017).

[77] G. Papallo, On the hyperbolicity of the most general Horndeski theory, Phys. Rev. D 96, 124036 (2017).

[78] F.-L. Julié and E. Berti, $d+1$ formalism in Einstein-scalarGauss-Bonnet gravity, Phys. Rev. D 101, 124045 (2020).

[79] H. Witek, L. Gualtieri, and P. Pani, Towards numerical relativity in scalar Gauss-Bonnet gravity: $3+1$ decomposition beyond the small-coupling limit, Phys. Rev. D 101, 124055 (2020).

[80] W. E. East and J. L. Ripley, Evolution of Einstein-scalarGauss-Bonnet gravity using a modified harmonic formulation, Phys. Rev. D 103, 044040 (2021). 